\title{
Large-Scale Production of Nanocrystalline Black
}

\section{Phosphorus Ceramics}

Nikolas Antonatos ${ }^{1}$, Daniel Bouša ${ }^{1}$, Evgeniya Kovalska ${ }^{1}$, David Sedmidubský ${ }^{1}$, Květoslav

Růžička ${ }^{2}$, Pavel Vrbka ${ }^{2}$, Martin Veselý' ${ }^{3}$ Jiři Hejtmánek ${ }^{4}$, Zdeněk Sofer ${ }^{1, *}$

${ }^{1}$ Department of Inorganic Chemistry, University of Chemistry and Technology Prague,

Technická 5, 16628 Prague 6, Czech Republic; zdenek.sofer@vscht.cz; Fax: +420

220440422

${ }^{2}$ Department of Physical Chemistry, University of Chemistry and Technology Prague, Technická 5, 16628 Prague 6, Czech Republic

${ }^{3}$ Department of Organic Technology, University of Chemistry and Technology Prague,

Technická 5, 16628 Prague 6, Czech Republic

${ }^{4}$ Institute of Physics of the Czech Academy of Sciences, v.v.i., Cukrovarnická 112/10, 16200

Prague 6, Czech Republic

\section{Corresponding Author:}

*Zdeněk Sofer, Department of Inorganic Chemistry, University of Chemistry and Technology Prague, Technická 5, 16628 Prague 6, Czech Republic. E-mail: zdenek.sofer@vscht.cz. 


\section{$\mathrm{P}$ K $\alpha 1$}
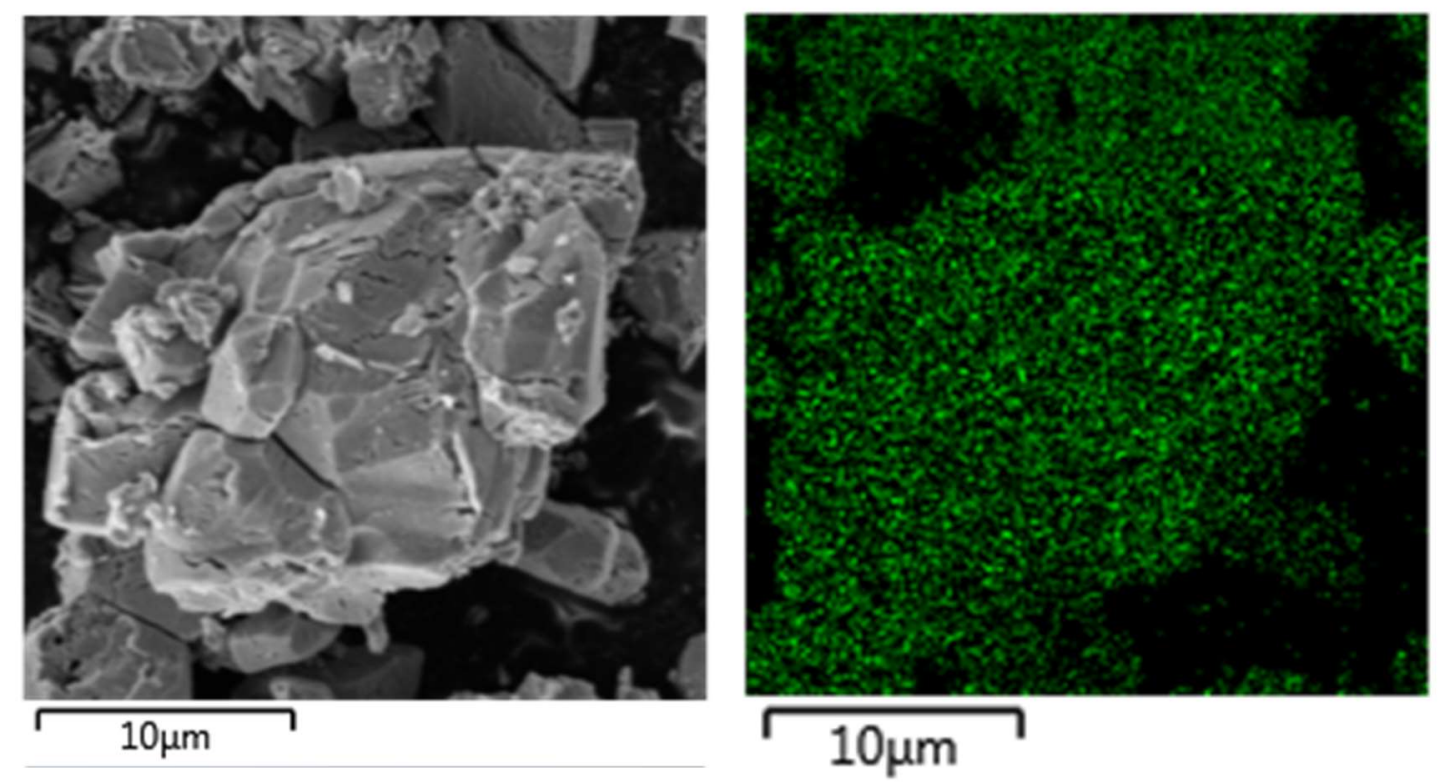

Figure S1. EDS images and the corresponding phosphorus elemental map of black phosphorus ceramic showing the uniform distribution of the element on the material.

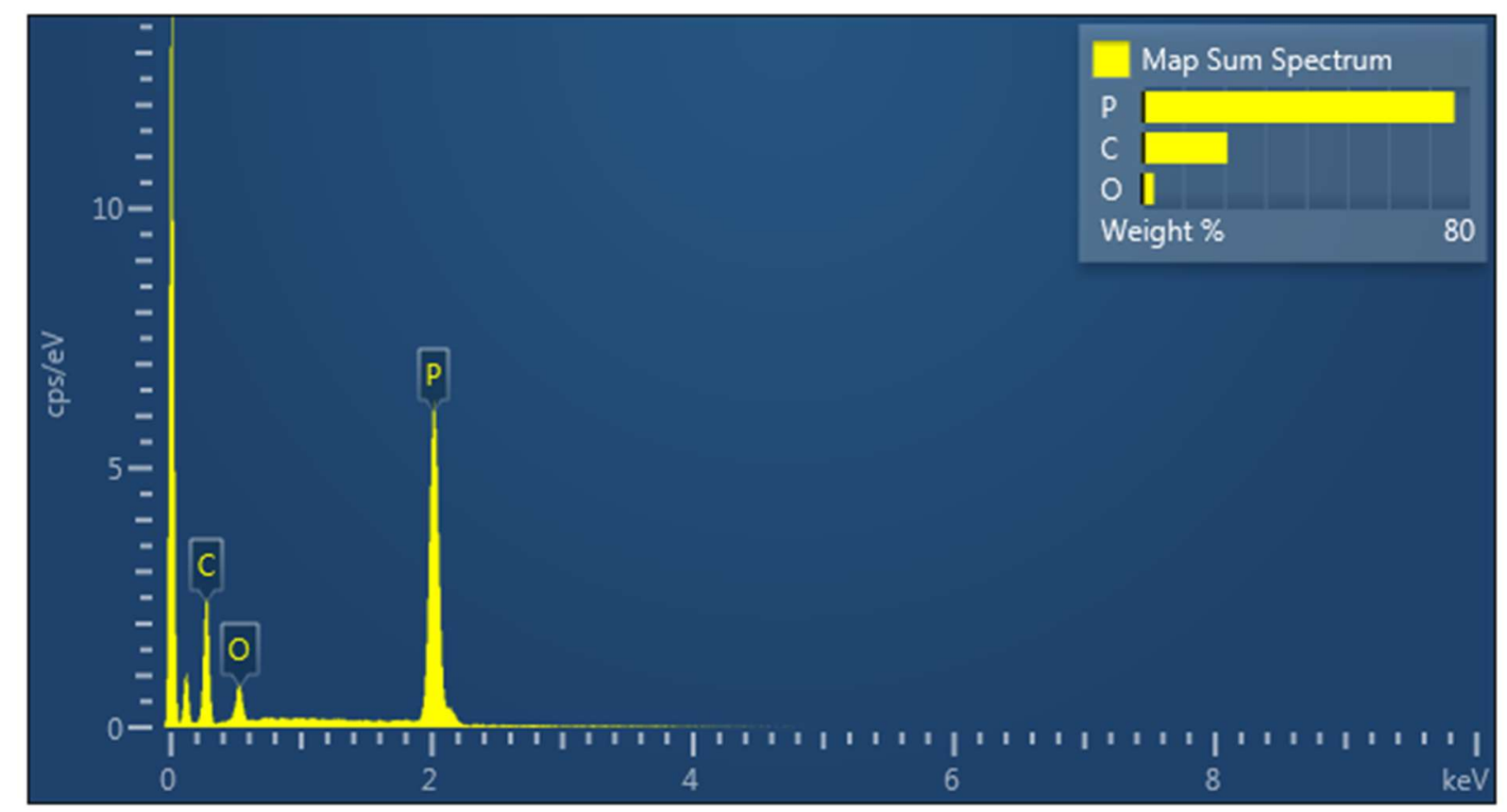

Figure S2 EDS of the atomic elemental percentage of black phosphorus ceramic showing no additional impurities were observed in the material. 


\section{$\mathrm{P} \mathrm{K} \alpha 1$}
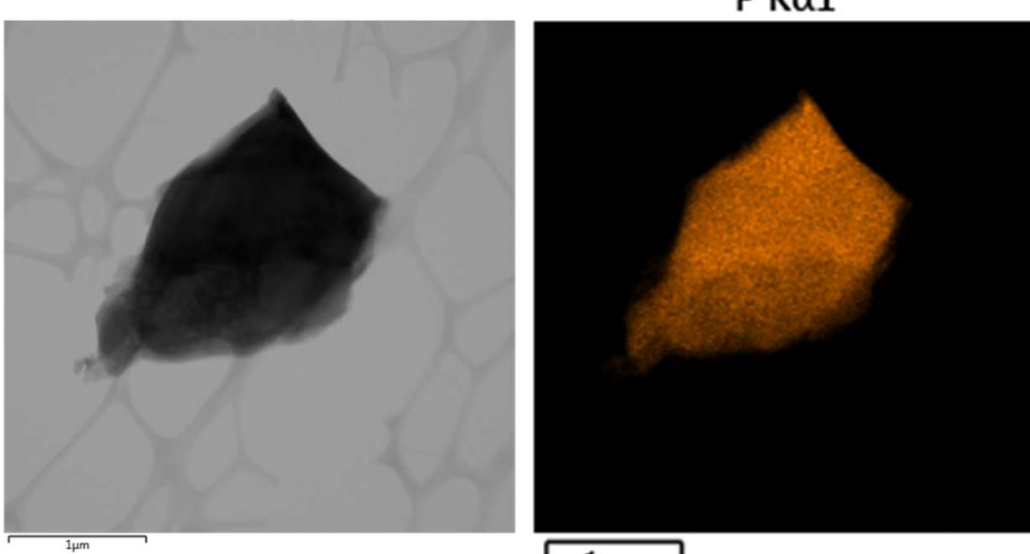

$1 \mu \mathrm{m}$
$\mathrm{O} K \alpha 1$

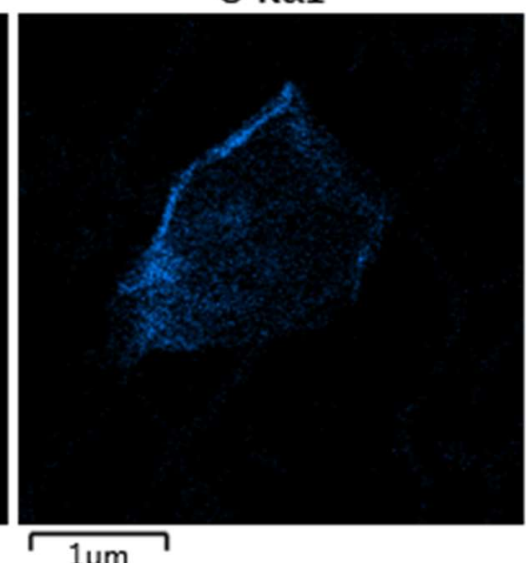

$1 \mu \mathrm{m}$

Figure S3. TEM/EDS images of black phosphorus ceramic depicting the uniform distribution of $\mathrm{P}$ in the material while oxidation of the material is only local.

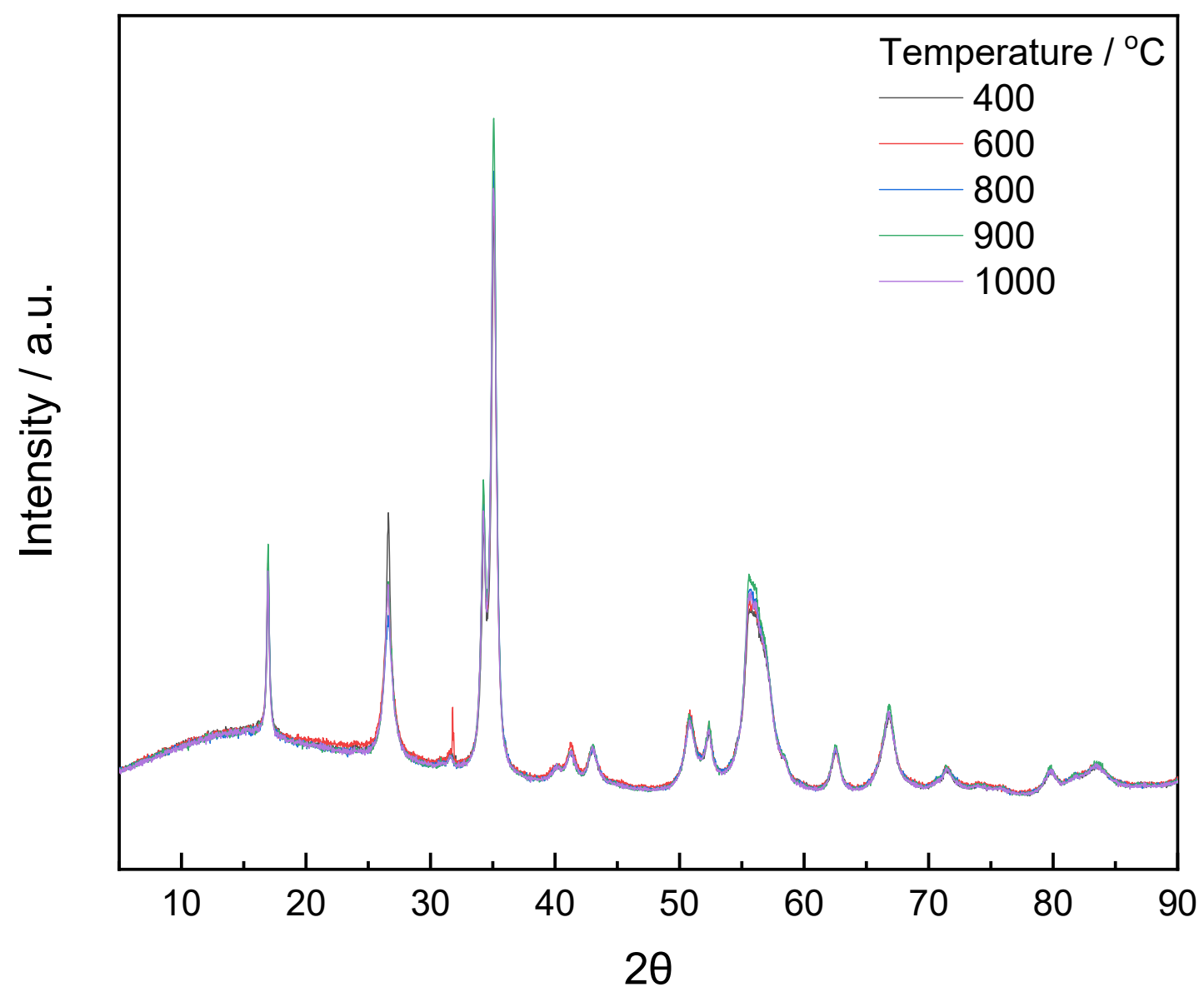

Figure S4. X-ray diffractograms of black phosphorus ceramics prepared at different temperatures. 
Table S1. Summary of the crystallite size and full-width half maximum of the two most intensive peaks obtained from X-ray diffraction analysis of black phosphorus ceramics prepared at different temperatures.

\begin{tabular}{|c|c|c|c|c|}
\hline \multirow{2}{*}{$\begin{array}{c}\text { Temperature of } \\
\text { synthesis } /{ }^{\circ} \mathrm{C}\end{array}$} & \multicolumn{2}{|c|}{$\begin{array}{c}(111) \text { face } \\
2 \theta=35.10^{\circ}\end{array}$} & \multicolumn{2}{|c|}{$\begin{array}{c}(021) \text { face } \\
2 \theta=35.10^{\circ}\end{array}$} \\
\hline & Crystallite size / nm & FWHM & Crystallite size / nm & FWHM \\
\hline 400 & 15.14 & 0.47 & 11.83 & 0.54 \\
\hline 600 & 14.78 & 0.50 & 8.17 & 0.76 \\
\hline 800 & 14.13 & 0.51 & 7.55 & 0.84 \\
\hline 900 & 15.39 & 0.47 & 9.48 & 0.66 \\
\hline 1000 & 14.51 & 0.49 & 9.27 & 0.67 \\
\hline
\end{tabular}

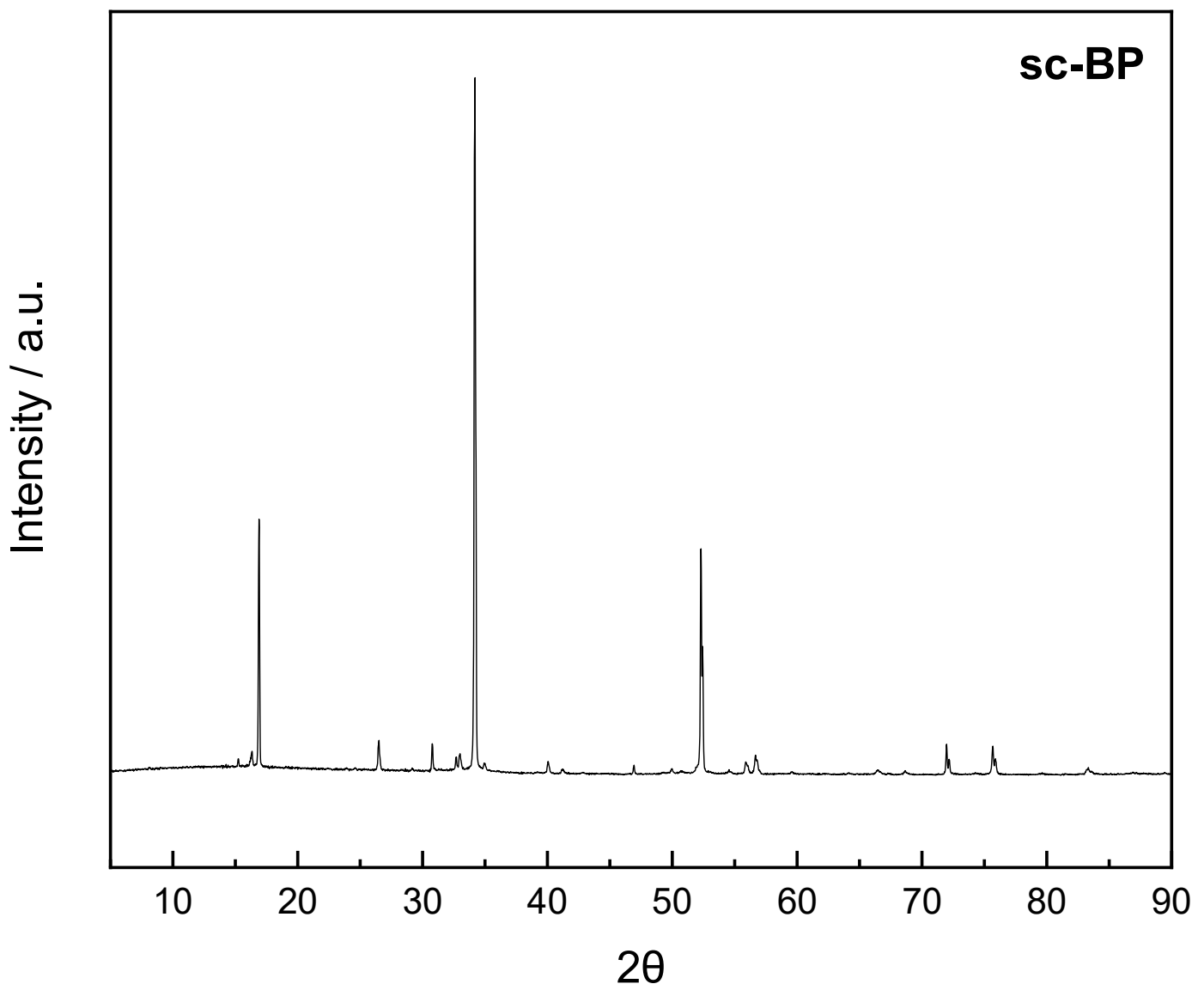

Figure S5. XRD pattern of single crystal black phosphorus. 


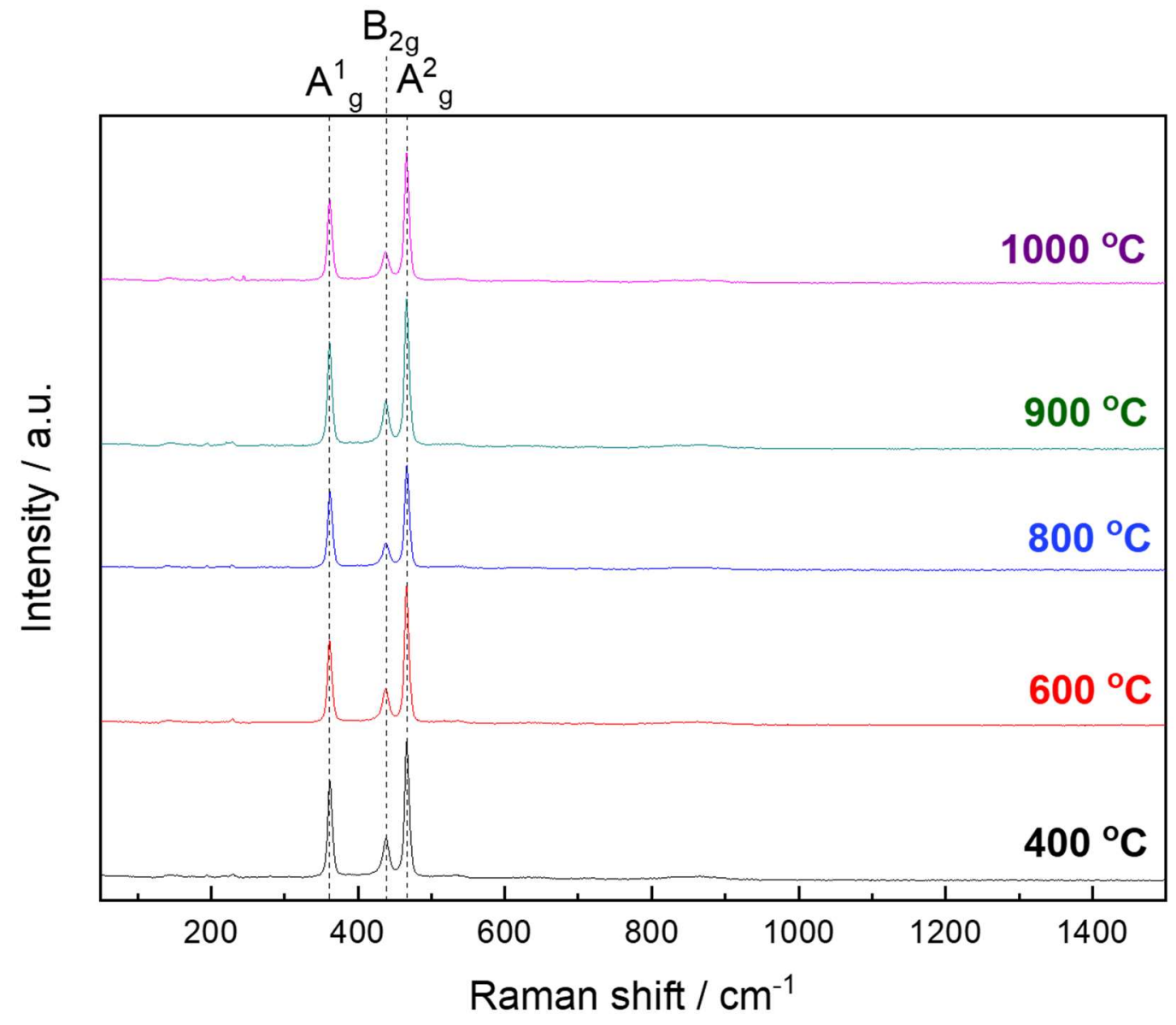

Figure S6. Raman spectra of black phosphorus ceramics prepared at different temperatures.

\section{Combustion enthalpy measurement}

An equation for the Washburn correction was derived in the same manner as described in literature ${ }^{1}$. It was assumed that the gas phase in the dry bomb consists only of pure oxygen and the volume of the phosphorus sample and the solid combustion product $\left(\mathrm{P}_{4} \mathrm{O}_{10}\right)$ were neglected. The amount of oxygen in the bomb was calculated using equations taken from literature ${ }^{1}$ and for the correction a final equation was derived.

$-\Delta U_{\text {Wash }}=n_{1} 0.0663\left(p_{1}-1\right)+n_{2} 0.0663\left(1-p_{2}\right)$

where $n_{1}, n_{2}$ and $p_{1}, p_{2}$ is the amount and pressure of oxygen in the bomb before and after the combustion, respectively. The parameter 0.0663 is the simplifying factor taken from literature ${ }^{1}$ giving an energy dimension of $11 \mathrm{~atm}$. Afterwards, the following conversion factors were used for recalculations:

\footnotetext{
${ }^{1}$ Washburn, E.W., J. Res. Nat. Bur. Stand., 1933, 10, 525
} 
$11 \mathrm{~atm}=24.2 \mathrm{cal}, 1 \mathrm{cal}=4.1868 \mathrm{~J}$, molar mass of phosphorus $123.896 \mathrm{~g} \mathrm{~mol}^{-1}$.

Table S2. Experimentally determined values of combustion enthalpy of red and black phosphorus together with the reported value of white phosphorus.

\begin{tabular}{|c|c|c|c|c|c|c|}
\hline Sample & $m / g$ & $Q_{\text {total }} / \mathrm{J}$ & $Q_{\text {total }}-Q_{\mathrm{el}} / \mathrm{J}$ & $8 U_{\text {Wash }} / \mathrm{J}$ & $\begin{array}{l}\Delta U_{\text {comb }}^{\mathrm{o}} / \\
\mathrm{kJ} \mathrm{mol}{ }^{-1}\left(\mathrm{P}_{4}\right)\end{array}$ & $\begin{array}{l}\Delta H^{\mathrm{o}} \text { comb } \\
\mathrm{kJ} / \mathrm{mol}^{-1}\left(\mathrm{P}_{4}\right)\end{array}$ \\
\hline white $^{2}$ & - & - & & & $\begin{array}{l}-2973.5 \\
\pm 4.0\end{array}$ & $\begin{array}{l}-2985.9 \\
\pm 4.0\end{array}$ \\
\hline red & 0.3292 & 8222.0 & 7906.2 & -5.13 & -2977.5 & -2989.6 \\
\hline red & 0.2089 & 5286.6 & 4970.7 & -3.27 & -2950.0 & -2962.2 \\
\hline red & 0.3746 & 9262.6 & 8946.8 & -5.83 & -2961.0 & -2973.2 \\
\hline red & 0.4257 & 10576.8 & 10261.0 & -6.60 & -2988.3 & -3000.5 \\
\hline red & 0.4875 & 11891.2 & 11575.4 & -7.54 & -2943.8 & -2955.9 \\
\hline black & 0.3539 & 8578.2 & 8262.4 & -5.51 & -2894.5 & -2906.7 \\
\hline black & 0.3367 & 8081.9 & 7766.1 & -5.25 & -2859.6 & -2871.8 \\
\hline
\end{tabular}

m...sample mass

$Q_{\text {total....total heat produced at combustion experiment }}$

$Q_{\mathrm{el} . \ldots}$ heat evolved by electric energy and by iron wire combustion $(316 \pm 16) \mathrm{J}$

$8 U_{\text {Wash... Washburn correction }}$

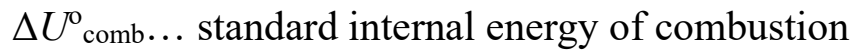

$\Delta H^{\mathrm{o}}$ comb... standard enthalpy of combustion

\footnotetext{
${ }^{2}$ Holmes, W.S., Trans. Faraday Soc., 1962, 58, 1916
} 


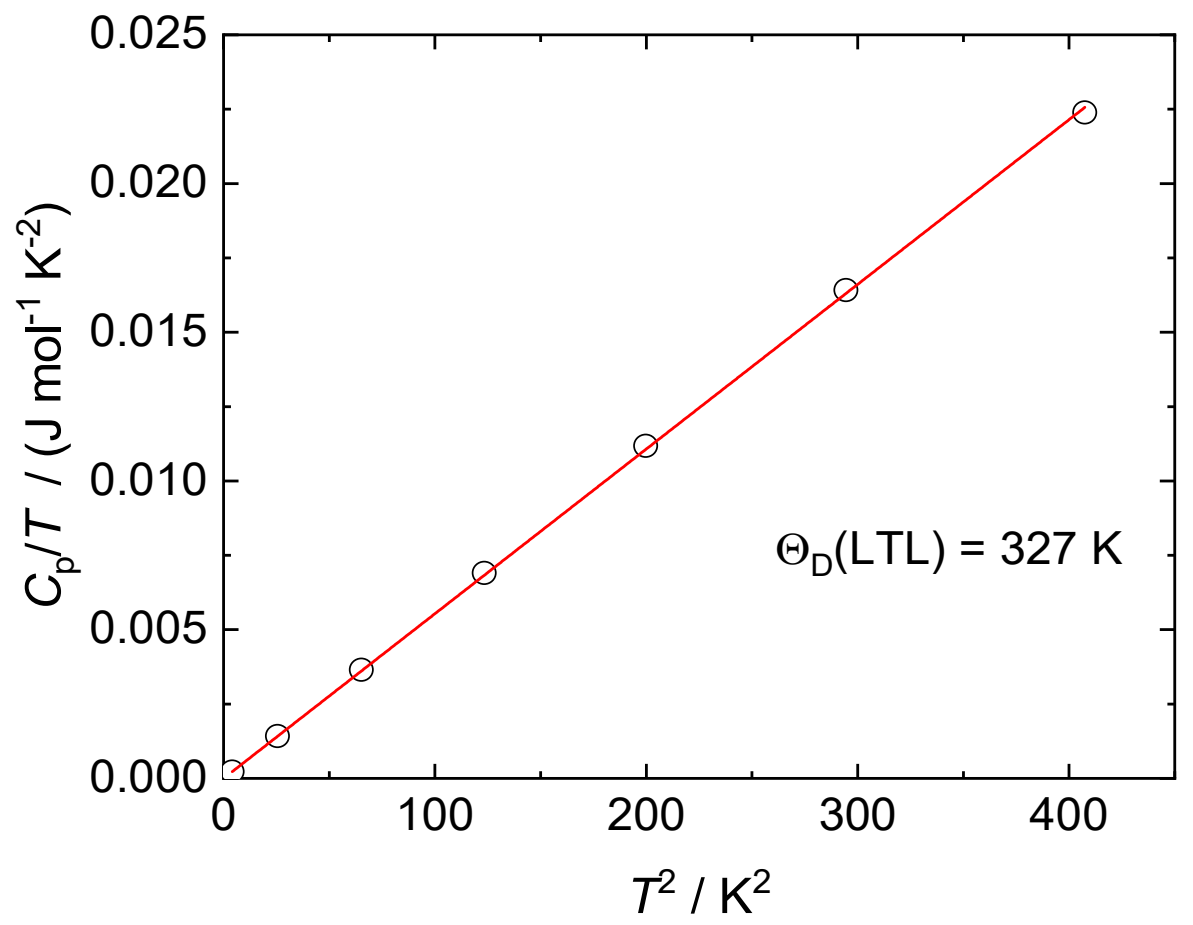

Figure S7. Low temperature limit of heat capacity in the $C_{p} / T$ vs. $T^{2}$ representation. The slope of the linear dependence determines the apparent Debye temperature.

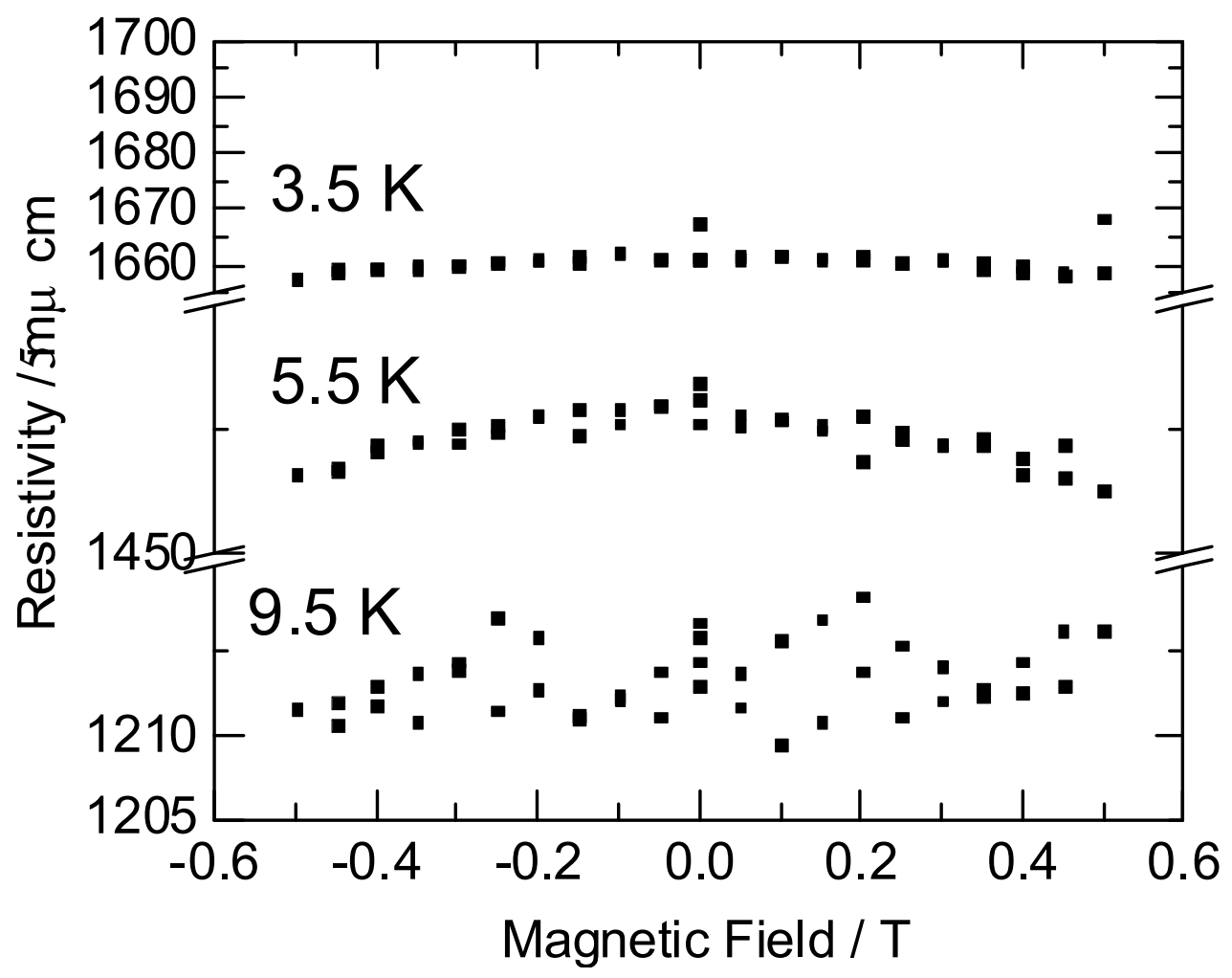

Figure S8. Magnetoresistivity of black phosphorus ceramic at low temperatures in a $\pm 0.5 \mathrm{~T}$ magnetic field. 


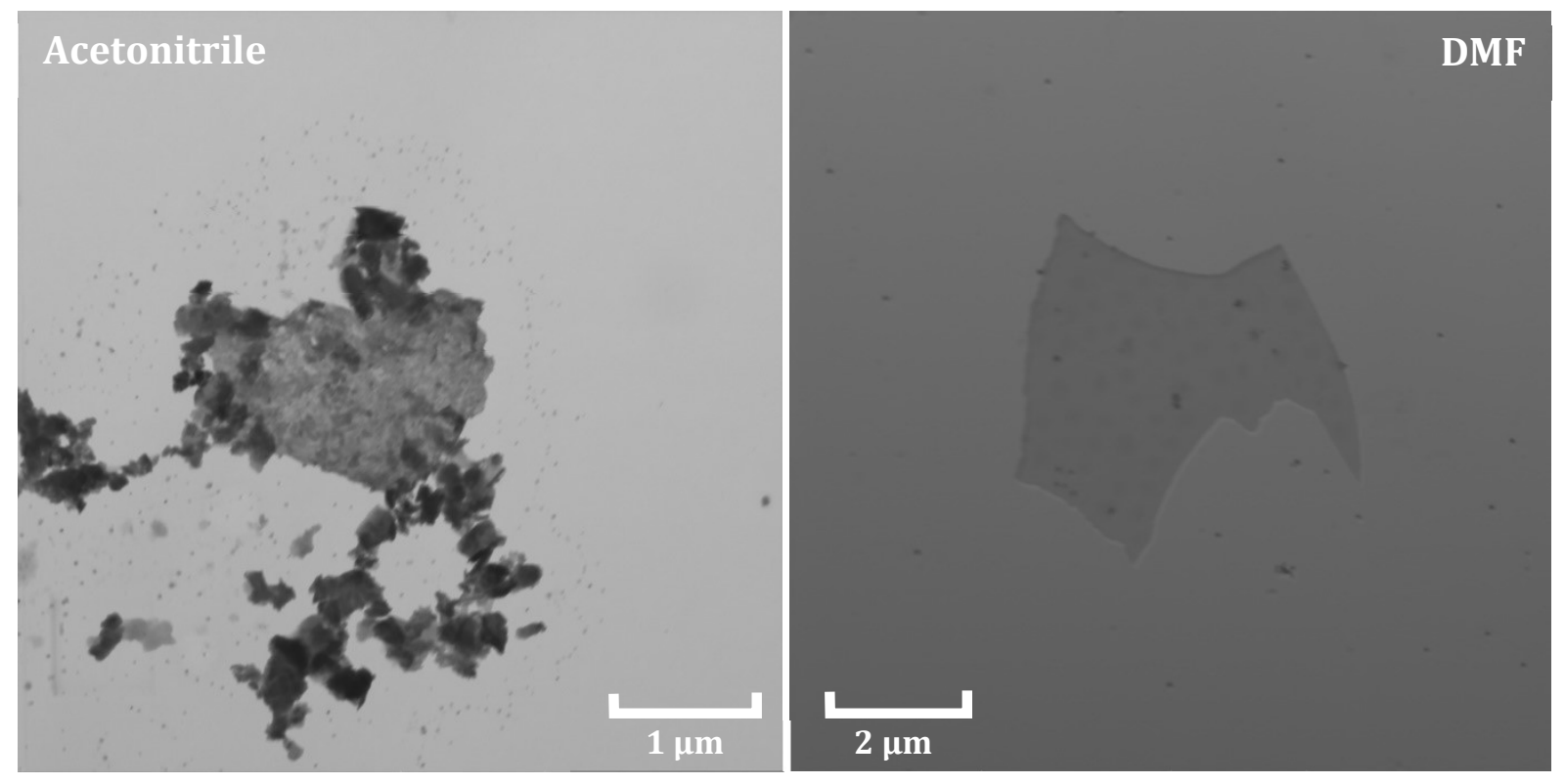

Figure S9. STEM images of black phosphorus nanoparticles after exfoliation for $120 \mathrm{~min}$ of milling time in acetonitrile (left) and DMF (right).

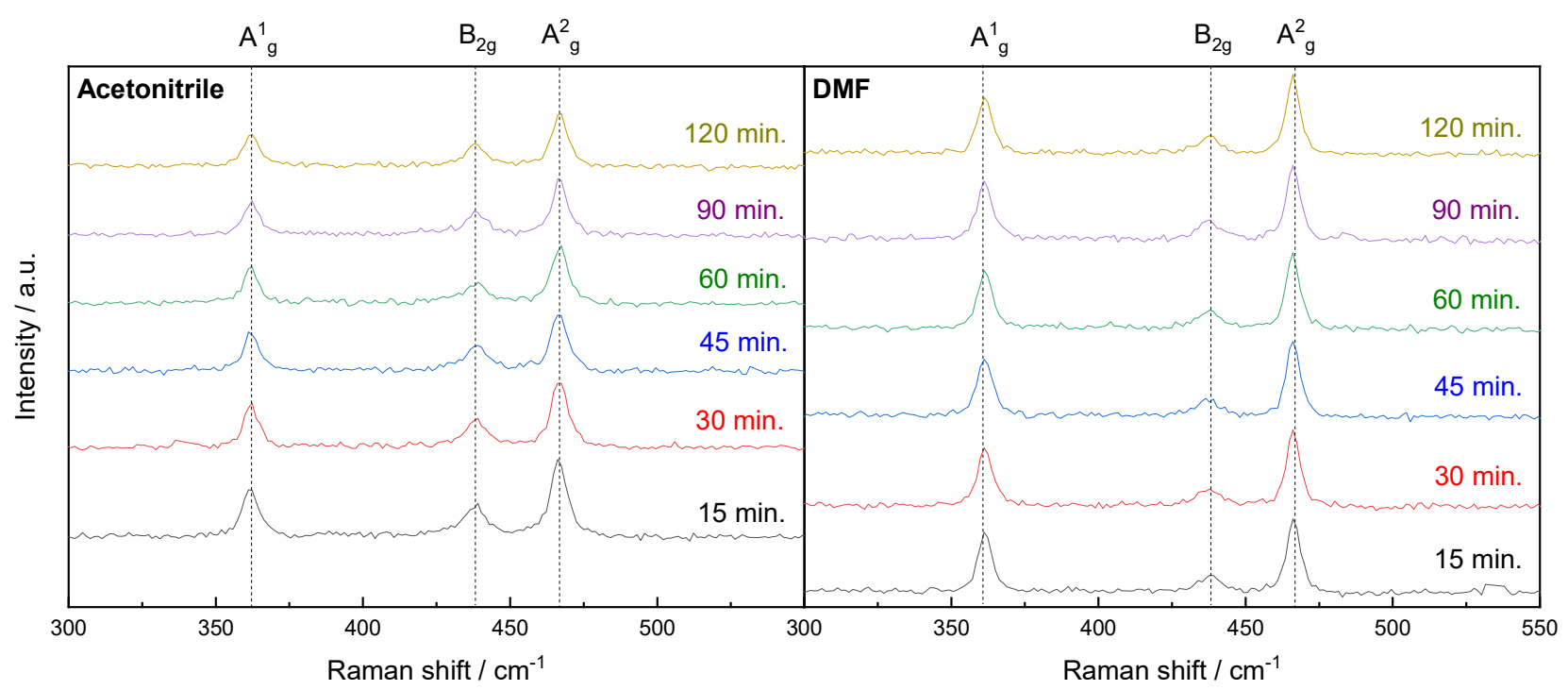

Figure S10. Raman spectra of exfoliated BP ceramics in acetonitrile (left) and DMF (right).

The time refers to milling time during exfoliation. 
a)

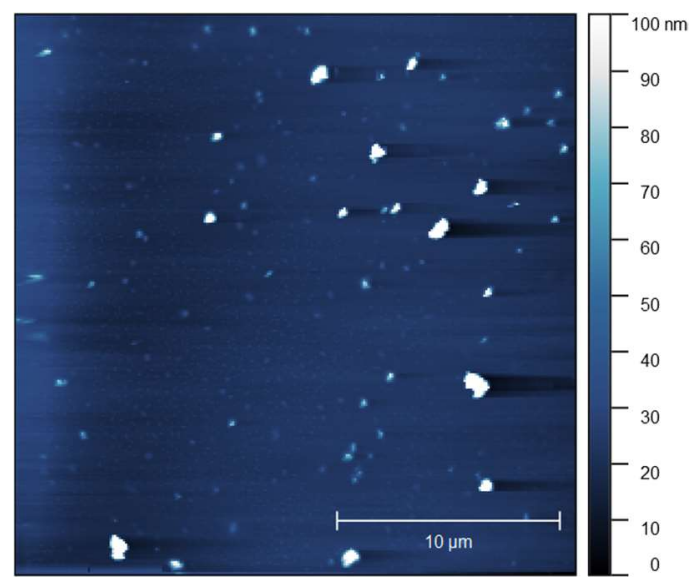

c)

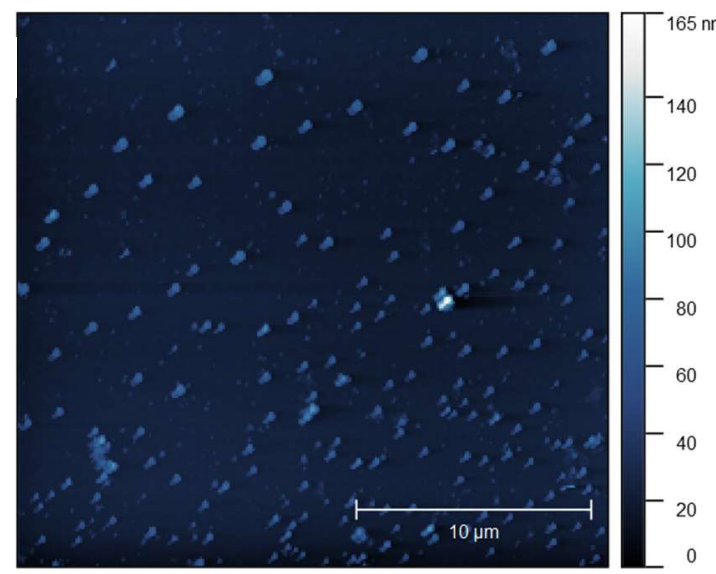

b)

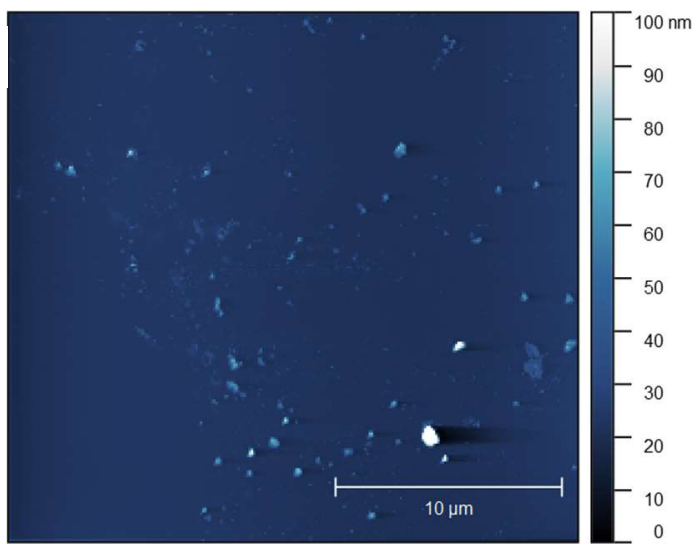

d)

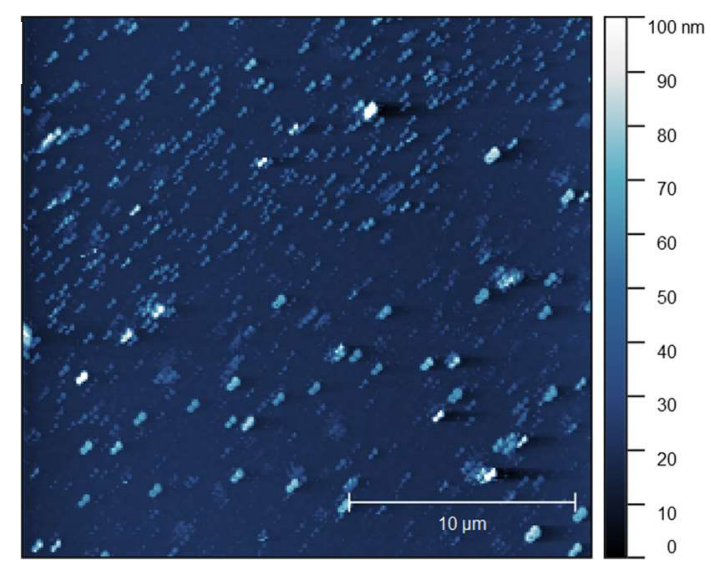

Figure S11. AFM images of exfoliated BP ceramics in acetonitrile after a) 60 and b) 120 minutes and in DMF after c) 60 and d) 120 minutes. 

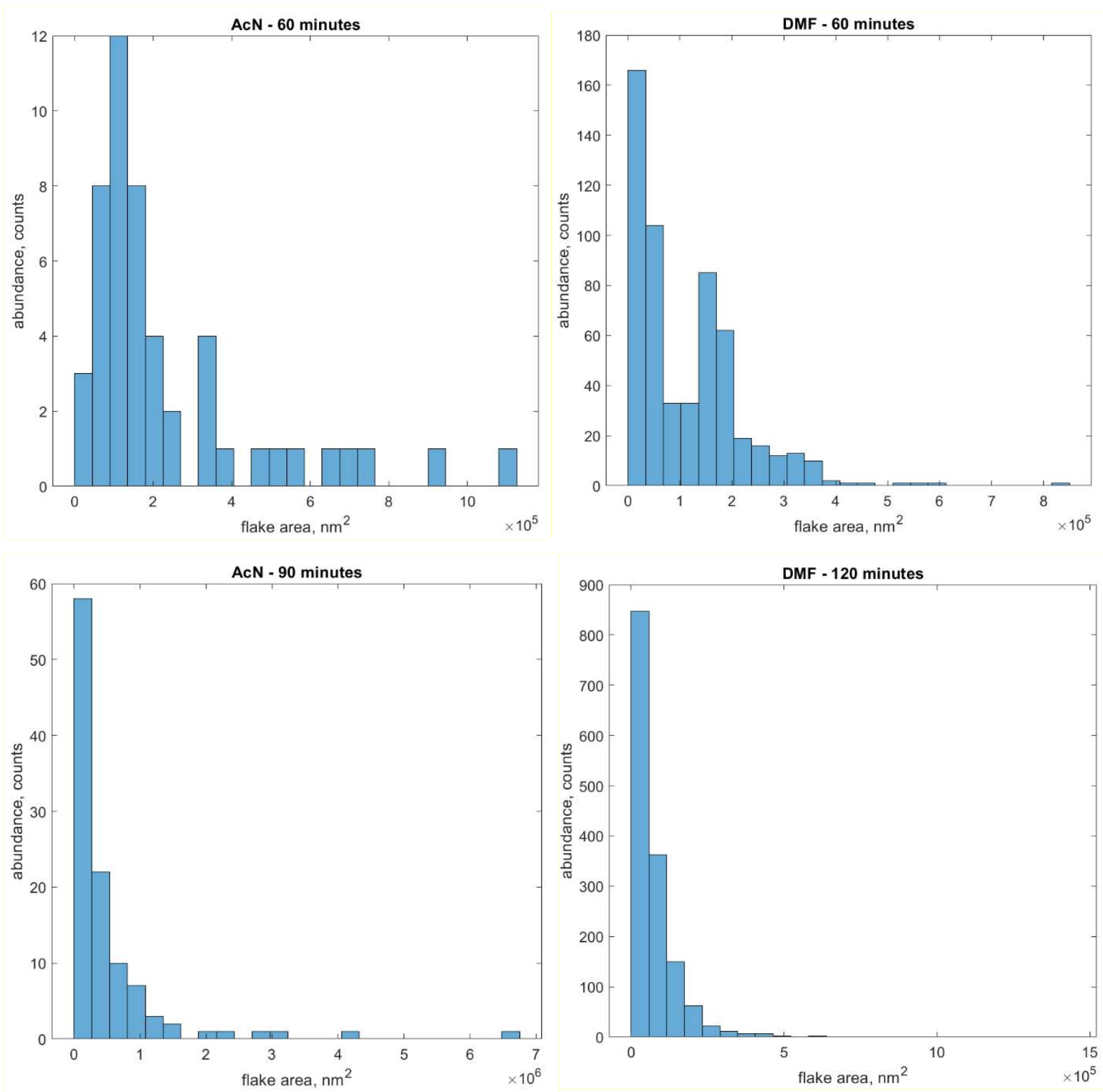

Figure S12. Particle size distribution of exfoliated BP ceramics in acetonitrile and DMF. The milling time and solvent used is labelled above each graph. 